\title{
A real-time man-in-loop threat evaluation and resource assignment in defense
}

\author{
Afshan Naseem ${ }^{1,2 *}$, Shoab Ahmed Khan ${ }^{1,2}$ and Asad Waqar Malik ${ }^{3}$ \\ ${ }^{1}$ Center for Advanced Studies in Engineering (CASE), Islamabad, University of Engineering and Technology \\ (UET), Taxila, Pakistan; ${ }^{2}$ College of Electrical and Mechanical Engineering (CEME), National University of \\ Sciences and Technology (NUST), Rawalpindi, Pakistan; and ${ }^{3}$ School of Electrical Engineering and Computer \\ Sciences (SEECS), National University of Sciences and Technology (NUST), Islamabad, Pakistan
}

Efficient weapon threat assignment reflects military proficiency and requires prompt decision while managing the available resources. An important problem which commanders/decision makers face is to optimally utilize the resources in complex and time constraints situations. Several solutions have been proposed in the literature. In this paper, an innovative approach is proposed for threat evaluation and weapon assignment (TEWA) by following 3-dimensional stable marriage algorithm (3-D SMA). This proposed model incorporates new parameters and constraints i.e. supply chain, inventory of resources and multiple threats-weapons assignments that outperforms the previous techniques. This suggested model is based on threat perception followed by an integration of parametric based automatic threat evaluation technique for further weapon scheduling and assignment problem keeping in view that the threat with greater threat index has higher priority to be intercepted and weapons' kill probability. The experimental section shows that our proposed approach has greatly improved in comparison with other approaches. The results showed that the threat neutralization is improved up to $25 \%$ reducing the usage of ammunition till $31.1 \%$. The damage of assets abridged to $28.5 \%$ in comparison with existing approaches. The proposed approach elucidates that TEWA is an efficient real-time threat perception and optimal multi-threat scheduling problem at weapons' resolution. It is a three-stage process, where the first stage perceives the threat, the second stage works on threat evaluation and the final stage focuses on weapon scheduling and assignment problem. The addition of new parameters and constraints in the new proposed model makes it a unique approach in which more accurate results, in neutralizing the threats, are obtained with less use of ammunition and damage of assets that makes TEWA more effective and efficient tool for optimum decision making in time critical situations.

Journal of the Operational Research Society (2017) 68(6), 725-738. doi:10.1057/s41274-016-0139-6; published online 16 December 2016

Keywords: threat perception; evaluation; weapon assignment; stable marriage algorithm; threat index; supply chain; inventory

\section{List of symbols}

$\mathrm{VA}_{j} \quad$ Set of vulnerable assets

$e \quad$ Total number of vulnerable assets

$T_{n} \quad$ Set of threats

$F \quad$ Total number of threats

$u \quad$ Weight assigned to parameters

$v \quad$ Weight assigned to parameters

kp Kill probability

$T(\mathrm{~d}) \quad$ Distance of threat from asset

$T$ (ht) Height of threat

$T(\mathrm{~h}) \quad$ Heading vector of threat

$T(\mathrm{~m}) \quad$ Maneuvering of threat

$T$ (f) Threat formation

T(1) Threat lethality

*Correspondence: Afshan Naseem, Center for Advanced Studies in Engineering (CASE), Islamabad, University of Engineering and Technology (UET), Taxila, Pakistan.

E-mail: afshan_naseem@yahoo.com; afshan.naseem@ceme.nust.edu.pk
$T_{\mathrm{v}} \quad$ Threshold value

$\mathrm{AT}_{i} \quad$ Approaching time of a specific threat

$V_{i} \quad$ Velocity of approaching threat

II Intent index of a threat

CI Capability index of a threat

$W_{\mathrm{k}} \quad$ Set of weapons

$m \quad$ Total number of weapons

$Q_{\text {s }} \quad$ Set of maximum suitable available weapons

TI( $i) \quad$ Threat index value of threat $i$

DW Deployed weapons

WS Scheduling of weapons

ME Multiple engagements of weapons

MWA Multiple weapon assignment of weapons

Ld Load on deployed weapons

LT Threshold of a weapon

$\Delta t \quad$ Least possible time required to engage successive threats

rlt Reload time (weapon) 
stb Stabilization time (weapon)

mov Time entails to move from one threat POE to another threats POE

WIC Weapon/ammunition in inventory check

\section{Introduction}

Strategic cognitive decision making is considered one of the most crucial activities in real time environment of military defense. The constraints associated with the assessment and evaluation of threats to come up with optimum solution, exist within the framework of available resources, technological advancement and impending uncertainties (Azimirad and Haddadnia, 2015; Erlandsson and Niklasson, 2014). To suggest an appropriate approach, many researchers have studied threat assessment (Azimirad and Haddadnia, 2015; Wu and Ye, 2014; Riveiro et al, 2014; Dongfeng et al, 2012; Oxenham, 2003; Nguyen, 2002; Liebhaber and Feher, 2002; Roy et al, 2002), however, the decision making while assessing the aerial threat and assigning the most effective weapon is still an open issue.

Threat evaluation and weapon assignment (TEWA) is an intricate system whose verdicts are based on numerous factors and cannot be demarcated as a separate model; they are interwoven with each other. TEWA occupies core components of an Air Defense (AD) system (Riveiro et al, 2014). Manual Threat Evaluation (TE) is always challenging especially in serious situations (Riveiro et al, 2013). In even semiautomated mode, operators' input is used in computerized decision support system (Roux and Vuuren, 2007). Most of the time, there are critical instances where rapid decisions are required for effective neutralization of threats and protection of vulnerable areas or vulnerable points (VA/VPs) (Barkdoll et al, 2002), which makes the task of operators extremely important primarily because efficient weapon selection highly depends upon observer's input (Allouche, 2005). In these decisive situations, inevitably momentous pressure develops on the mind of observer that may disturb the TE process. A misclassification may result in jeopardizing the whole system. Realizing this, many countries are now working on their Net Centric Programs and with much emphasis on AD system. Most of the net centric AD systems work on automated or semi-automated TEWA systems. Manual TEWA cannot deliver optimality because of numerous factors that come into play with respect to the observer. These factors are comprised of information, inadequate awareness, state of mind, visibility and human error etc. As a result, in real circumstances, defense system failed to produce optimal effectiveness (Beare, 1987; Nguyen et al, 1997; Kirby and Capey, 1997). On one side, it causes loss of expensive ammunitions and on the other, it fails to protect the VA/VPs which appears to be an important constraint of TEWA in real-time decision making process (Dongfeng et al, 2012).

Most of the solutions to TEWA use static or 2-dimensional (2-D) approaches, neglecting few important parameters. In this paper we presented an efficient TEWA model paring real-time threat perception (TP) and threat-weapon to cater many-to-many relationship keeping in mind that TEWA in $\mathrm{AD}$ is a 3-dimensional (3-D) problem. Our proposed solution uses a variant of stable marriage algorithm (SMA). It includes knowledge-base to support identification of threat intent and capability along with weapon assignment (WA) in time-critical situations. The section II of this paper is an overview of TEWA and SMA. The proposed TP and WA model is defined in Section III. Section IV describes experimental setup, results and comparison with an existing approach.

\section{TEWA and previous approaches}

TEWA is a multifaceted system. Its foremost objective is to proficiently evaluate threat and then assign weapon understanding the intent, objectives and capacities of the enemy's attack (Hausken and Zhuang, 2012). It is used for optimization of available resources. There are two phases of this dynamic system with complex and ever-changing environment. First phase is threat perception, evaluation and prioritization of threats. TE is a stimulating task that comprised of multiple parameters and processes (Govindan et al, 2015; Sahin and Leblebicioglu, 2014). These parameters are measured to provide threat indexing for combat concept (Azimirad and Haddadnia, 2015). TE is a key phase of TEWA. It can be made more effective by using multiple weighting utility function. The different weights can be assigned to different characteristics depending on the function of range from threat to a VA/VP (Truter, 2016). Ascertaining an erroneous threat would cause system failure like many years ago, 1980, a misclassification of non-threat as a threat caused a shoot down of an Iranian commercial airline (Liebhaber and Feher, 2002).

The threat of Weapons of Mass Destruction (WMD i.e., biological, chemical, radiological, high explosive and nuclear weapon) is an urgent and important threat for any country (Sahoo, 2016). The effect of attacks especially WMD can be mitigated with early preparation and disrupting the sabotage supply chain. The supply chain is an important factor in 
optimization problem of threats and defender game. Furthermore, the decision of subsidization depends on the preparation and attacking cost (Shan and Zhuang, 2014). The countermeasures can increase the cost-effectiveness and decrease the loss to the defender (Paulson et al, 2016). The threats are arranged based on threat index (TI). In most of the previous work, threats are evaluated based on intent and capability. The former refers to the willingness to hit VA/VPs while later states the ability of threat to cause damage. Both capability and intent models have their implications (Paradis et al, 2005), but combining both models can give an effective TE model, i.e. opportunity model (Naeem and Masood, 2010). The sequential game is another an effective technique for complete and incomplete information model for threats with inclusion of sensitivities of equilibria as a function combining the threat and defender's optimization problem. Although, it maximizes the expected utility, but the functional forms in this technique are simplistic and requires to add more complex function (He and Zhuang, 2012). The sequential game technique is applied on WMD attacks assuming the perfect and complete information encompassing the attacking cost analysis. It has been observed that the preparation cost is higher for WMD attack as compared to regular attack (Shan and Zhuang, 2014).

In calculation of TI, many factors are used. Threat stabilization is one of the significant factor associated with maneuvering situations (Allouche, 2005). This methodology is based on Kohonen's self-organizing maps. When the threat is maneuvering, it converts extremely difficult to envisage threat destination (Erlandsson and Niklasson, 2013; Phillips-Wren et al, 2009). There are different techniques used for TI like Bayesian networks and fuzzy logic (Mardani et al, 2015; Liao, 2000). Bayesian network approach uses the mathematical foundation and have more accurate values in uncertainties (French, 2015; Johnston et al, 2006; Cavalcante et al, 2010) but demand more development time as compared to fuzzy logic. TE can be calculated effectively using a fuzzy rule-based inference method. Fuzzy inference is considered an appropriate method integration of various threat characteristics and quantifying level of threat (Choi et al, 2016). In fuzzy logic, uncertainty is difficult to handle (Lozano et al, 2015). The hybrid approach is more effective in optimality (Johansson and Falkman 2008a, b). The game-theory is one of the techniques that helps in solving complex problem with existence of uncertainties, short reaction time and huge amount of data (Shan and Zhuang, 2013). The optimal resource allocation can be done with the combination of game-theory with multiattribute utility model depending on the information about threat and weapon. This model assumes that attacker has all the information of defender's resource allocation (Paulson et al, 2016) while in real-time environment, it is not necessary in all cases that the attacker has the detail facts of defender's resources (Hausken and Zhuang, 2012).

The cost is not limited to only monetary terms when security is a perilous factor (Li et al, 2012; Yildirim et al, 2009; Masud et al, 1995). The cost-effective results are always desirous in every field. The objective of any country is to maximize the security of assets with minimization of cost. When the defenders are uncertain about the accurate information of attacks, the optimal defense may deviate from optimal value (He and Zhuang, 2012). The unit attack cost and unit defense costs are important parameters to consider in security. The dynamics of assets' valuation results in repeated attacks and the unit attack cost gradually decreases in repeated attacks (Hausken and Zhuang, 2012). The optimal solution can be achieved using objective function considering the budget and neuroscientific constraints (Onay, 2016).

Once threats are prioritized based on their TI values, they need to be neutralized with assignment of best available weapon. This come in the second phase of TEWA. WA is a complex process encompassing many related parameters. WA problem uniquely pairs the intent and capability aspects of threat while seeking minimization of survival probability of threats (Truter, 2016). Considering the complexity of WA problem and keeping in view its constraints, commanders are facilitated with fuzzy decision making to make quick and effective decisions (Liebhaber and Feher, 2002). TE facilitates the process of weapon assignment with intelligence sensor support system (Azimirad and Haddadnia, 2015; Erlandsson and Niklasson, 2013). Considering TEWA a complex realtime system with uncertainties and constraints, there are different approaches being used for WA problem. A weapon system (WS) has n number of weapons of different types with their own elevation angle, lethality index, rate of fire, priority and field of fire etc. (Beare, 1987). The weapon is assigned to threat based on the above mentioned parameters.

The objective function use for WA process is dependent on the defense strategy the commander wants to adopt. In most of the cases, the commander wants to minimize the survival value of threats with minimization function and maximize the survival value of VA/VPs (Karasakal, 2008; Smith et al, 2001) with maximization function. The contest success function is used for the defender-attacker move considering the cost and time parameters to find the optimal solution. The repeated attacks drain the resources which results in increasing the unit attack cost (Hausken and Zhuang, 2012). Most of the time, two types of approaches are considered: preferential defense strategy and subtractive defense strategy. In preferential strategy, the model aims to destroy all the threats with high probability, enough ammunition and minimum damage to assets (Hossein and Athans, 1990). It seems infeasible from ammunition perspective. The subtractive defense strategy is inversely proportional to the number of threats targeting to VA/VPs. The hybrid of both these strategy is more suitable in TEWA systems (Naeem and Masood, 2010).

The pairing of threat and weapon can be done with different approaches. One of the model consists of pre-processing algorithm and reward-based mixed integer programming information was developed to use. Although this algorithm matches the threats with weapons based on profiles and capabilities, but this model is not quicker enough to produce 
efficient responses in multiple assignments in real-time environment (Laird, 2016). SMA is one of the effective approach that may find a best match between threat and weapon. This approach is based on matching between men and women for stable marriage, based on preference list. Many-to many SMA has more variations because it allows both men and women to have quota (Iwama, 2008). This approach is effective in dynamic systems. Although TEWA can be static or dynamic both, but with no doubt, dynamic TEWA is more effective as well as difficult to design and implement. Because, dynamic system facilitates the multiple engagements that is crucial in time-constraints environments (Hosein et al, 1988). Applying the approach of Many-to-many SMA on TEWA seems to provide remarkable results. Although, SMA has been applied on TEWA system but considering the 2-D parameters (Naeem and Masood, 2010). While, aerial TEWA is a 3-D problem and cannot be effectively solved with 2-D parameters.

Although many researchers have been working on improving TEWA systems, but with technological advancements, the need of improvement in the existing system is always there. Here we find a need to propose a 3-D SMA for effective TEWA system incorporating some missing constraints and parameters along with supply and inventory of weapons in TEWA system. In the next section, we propose an innovative approach for development of an effective and efficient TEWA system in $\mathrm{AD}$.

\section{Proposed approach}

The proposed solution to TEWA problem is a three phase process. In first phase, threats are perceived. In second phase, threats are evaluated and a TI is calculated for each threat based on TE and VA/VPs. The 3-D many-to-many SMA is used for pairing threats with VA/VPs. In third phase, WA is performed using dynamic weapon scheduling and paired with threats again using 3-D many-to-many SMA approach. In this proposed approach, few new parameters and constraints have been added those were missing in the previous studies. The parameters of 3-D interaction of threats and weapon selection incorporating inventory and supply chain of resources are added. The constraints of weapon selection and assignment encompassing multi-threat mapping with multi-weapon assignment are also mentioned in new approach. The addition of these new constraints and parameters make this approach an innovative one that produces more effective and efficient results in comparison with previous approaches.

Before going into the details of proposed solution, here we comprehend all the significant parameters that contribute in making decision using TEWA system (as shown in Table 1). Threats identification grounds on numerous parameters, which are comprised of threat height, speed, capability, radar crosssection, approach to vulnerable points/assets, maneuvering
Table 1 Critical parameters used for TEWA

\begin{tabular}{|c|c|}
\hline TEWA stages & Parameters \\
\hline \multirow{8}{*}{$\begin{array}{l}\text { Threat } \\
\text { perception }\end{array}$} & Height \\
\hline & Speed \\
\hline & Radar cross-section \\
\hline & Dive angle \\
\hline & Maneuvering capability \\
\hline & Attack approach \\
\hline & Thermal images \\
\hline & Heat signature \\
\hline \multirow[t]{10}{*}{ Threat index } & Distance from vulnerable points \\
\hline & Speed \\
\hline & Height \\
\hline & Altitude \\
\hline & Heading \\
\hline & Formation \\
\hline & Maneuver \\
\hline & Type \\
\hline & Carrying weapons \\
\hline & Threat lethality from knowledgebase \\
\hline \multirow{6}{*}{$\begin{array}{l}\text { Weapon } \\
\text { selection }\end{array}$} & Profile match to threat type \\
\hline & Capability \\
\hline & Kill probability \\
\hline & Range to neutralize threat \\
\hline & Quantity in inventory \\
\hline & Supply from depot (in warfare situations) \\
\hline \multirow{3}{*}{$\begin{array}{l}\text { Weapon } \\
\text { assignment }\end{array}$} & Weapon is assigned to a threat based on TI \\
\hline & $\begin{array}{l}\text { Weapon is assigned first to a threat with highest } \\
\text { TI (when threat is more than one) }\end{array}$ \\
\hline & $\begin{array}{l}\text { Weapon with highest kill probability is assigned } \\
\text { first }\end{array}$ \\
\hline
\end{tabular}

dive angle and etcetera. Utilizing vast number of parameters results in high precision outcomes of threat perception and evaluation. For correct TP, it is imperative to have an effective intelligent model originating from a knowledge-base which is actually an intelligent system that provides input for decision making. TI is calculated on the basis of threat identification. TI provides information for the purpose of ranking threats from most threatening to least one (Azimirad and Haddadnia, 2015; Tanergüçlü et al, 2012). In the present work, we have extended the process of TI calculation to several other factors to make the results more precise, accurate and comprehensive. These factors include threat formation, distance to nearest VA/VP, speed, threat capability/lethality, holding missiles, missile ranges, height, threat's profile and maneuvering capability (as shown in Table 1).

\subsection{Threat perception}

TP is certainly a multi-layer process model (Figure 1). It is used to assimilate TP component with other real-time applications. The Interface Module (IM) is used to facilitate interoperability amongst different modules. IM is specifically helpful in cross language/platform communication. IM performances as a conduit between Central Processing Module 


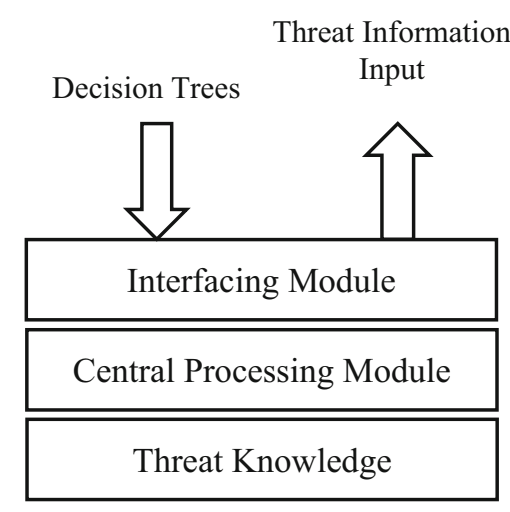

Figure 1 Threat perception model.

(CPM) and real-time applications. CPM is built using data mining algorithm for constructing the classification tree. The knowledge-base is directly linked with CPM module to support in classification tree. The threat cataloging is virtuously based on various parameters; among them radar cross section is one of the exceptionally important features. Radar cross section returns the size of cross section that is an indication of the material, relative size and incident angle of the target aircraft. This feature is especially used to generally categorize threat types i.e. Fighter, Transporter, Helicopter and etc. Radar cross section is a grouping of raw data and errors; threat maneuvering situations is exceedingly difficult to acquire an accurate radar cross section. Heat signature is another important factor to consider in threat information processing. Based on several other factors we developed a confidence level which indicates the level of trust on our TP model (Figure 1).

The confidence factor is a function of several parameters as listed here:

CF (Flight Plans, Heat Signature, Ceiling, Cross Section, Thermal Images, Speed).

Depending on the availability of sensor data, not all these factors may be available. The algorithm intelligently uses the available information in computing the TI.

It is observed the threat varies their height and speed especially in war situation. To cater this concern, a set of ranges are defined for classification based on fuzzy logic.

\subsection{Threat evaluation}

In our proposed TE strategy, we assume TP from different locations (radars/sensors). Once the information is provided, it is fused to create an amalgamated picture. Threat circumstantial information is used to measure distance of threat from all nearby approachable VA/VPs. These VA/VPs are chosen based on certain angle drawn from threat's heading.

The aim of TE is to evaluate threats on their capability and intent parameters and assign threats to VA/VPs that has Deployed Weapons (DW) to neutralize threats with maximum kill probability $(\mathrm{kp})$. Mathematically, the threats should be matched with VA/VPs.

$$
\begin{aligned}
& \mathrm{VA}=\left\{\mathrm{va}_{1}, \mathrm{va}_{2}, \mathrm{va}_{3}, \ldots, \mathrm{va}_{e}\right\} \\
& \text { where } e=\text { total number of vulnerable Assets } \\
& \text { and } T=\left\{t_{1}, t_{2}, t_{3}, \ldots, t_{f}\right\} \\
& \text { where } f=\text { total number of threats }
\end{aligned}
$$

The constraint applied to this pairing is:

$$
\sum_{g=1}^{e}\left(\text { Assigned }_{g, h}\right)=1 . \quad \text { where } h \in f, f=\{1,2, \ldots, F\} \text {. }
$$

The TI is calculated using the distance of a specific threat from targeted asset, its height, speed, heading, maneuvering, formation and lethality.

$\mathrm{TI}(i)=\{p \times(T(\mathrm{~d})+T(h t)+T(\mathrm{~h})+T(\mathrm{~m})+T(\mathrm{f}))+q \times(T(\mathrm{l}))\}$

Mathematical calculation of TI is based on the calculation of entry and exit points of threats in VA/VPs territory. The entry points show the possible points, a threat is expected to enter and exit points of threats to leave the territory.

This problem is dynamic and of 3-D nature. We need to make calculations for the aerial threats. In such circumstances, the territory of VA/VPs is represented with a sphere and a threat with straight line. Using sphere line intercept, points of threats intercept with VA/VPs circumference can be calculated as well.

$$
\left(x-x_{0}\right)^{2}+\left(y-y_{0}\right)^{2}+\left(z-z_{0}\right)^{2}=r^{2}
$$

where $C\left(x_{0}, y_{0}, z_{0}\right)$ is center of sphere representing VA/VPs and $r=$ radius of sphere.

Equations of straight line, representing threat, are:

$$
\frac{x-x 1}{l}=\frac{y-y 1}{m}=\frac{z-z 1}{n}=t
$$

where $t$ is the parameter.

$$
\text { or } \quad x=x_{1}+l t, \quad y=y_{1}+m t, \quad z=z_{1}+n t
$$

$l, m, n$ are direction cosines of straight line.

$$
l=\cos \alpha, \quad m=\cos \beta, \quad n=\cos \gamma
$$

where $\alpha, \beta, \gamma$ are direction angles representing threat's direction

$$
\begin{aligned}
& \text { and } \cos ^{2} \alpha+\cos ^{2} \beta+\cos ^{2} \gamma=1 \\
& \text { or } l^{2}+m^{2}+n^{2}=1
\end{aligned}
$$

Substituting (4) in (3)

$$
\left(x_{1}+l t-x_{0}\right)^{2}+\left(y_{1}+m t-y_{0}\right)^{2}+\left(z_{1}+n t-z_{0}\right)^{2}=r^{2}
$$


The values of $t$ (for threats) are given by the quadratic formula:

$$
t=\frac{-b \pm \sqrt{b^{2}-4 a c}}{2 a}
$$

where $a=1 ; \quad b=2\left(x_{1} l-x_{0} l+y_{1} m-y_{0} m+z_{1} n-z_{0} n\right.$; $c=\left(x_{1}-x_{0}\right)^{2}+\left(y_{1}-y_{0}\right)^{2}+\left(z_{1}-z_{0}\right)^{2}-r^{2}$

Putting values of $t$ in Eq. (4), we get points of intersection, i.e. entry and exit points of a threat $t_{\mathrm{k}}$ for a specific vulnerable asset. The entry point is the alert that provides the information about threat and the exit point is the last chance to neutralize the threat.

With values of $t$, other parameters like distance of threat and its approaching time (AT) can be calculated with simple formula of time, distance and speed.

$$
\mathrm{AT}_{i}=\frac{\left[T_{i}(d)\right]^{2}}{V_{i}}
$$

Threat lethality is calculated based on its profile and $\mathrm{kp}$. The $\mathrm{kp}$ shows the success probability of threat neutralization.

The numerator presents distance of threat from VA/VPs and denominator shows the threat's speed. The threat with lesser $\mathrm{AT}_{i}$ will be given higher intent value and final calculated TI will be greater using parametric equation. The threats are prioritized on the basis of their final TI.

Our proposed approach focuses on making the solution more robust and accurate by increasing the probabilities of success. $\mathrm{kp}$ is an important factor of success. Initial $\mathrm{kp}$ is calculated for all type of possible threats exist in database. $\mathrm{kp}$ shows the probability of achieving success in neutralizing threat when approaching to a particular VA/VPs. The $\mathrm{kp}$ is calculated using the intent and capability parameters of a threat. $p$ and $q$ are the weights assigned to threat parameters. The probability varies with changing the load on VA/VPs.

$$
\mathrm{kp}=1-(p \times \mathrm{II}+q \times \mathrm{CI})
$$

\subsection{Weapon selection and assignment}

The basic objective of WA is efficient utilization of ammunitions especially when multiple threats are moving toward the same asset. Weapon selection and assignment is a three-stage process. In the first step of WA phase, all candidate weapons are selected by taking into account current location and direction of threat. Reports have pointed out that the WA is mostly considered as single criterion decision problem (Lotter et al, 2013), however, in real terms it appears to be multi-objective decision problem encompassing cost of assigning weapon system and amassed survival probabilities of pragmatic threats. Once it is finalized and threats are perceived, then decision is made and weapon assignments are compiled.
Mathematically this problem is seen as a matching constraint where the elements of weapon should be matched with elements of threats.

$W=\left\{w_{1}, w_{2}, w_{3}, \ldots, w_{m}\right\}$,

where $m$ is total number of weapons

$T=\left\{t_{1}, t_{2}, t_{3}, \ldots, t_{n}\right\}$,

where $n$ is total number of threats

Subject to: $m<n$

$Q=\left\{q_{1}, q_{2}, q_{3}, \ldots, q_{q}\right\}$,

where $q$ is maximum suitable available weapons

The matching of threats and weapons is subject to the following constraint.

$$
\begin{aligned}
& \sum_{q=1}^{m}\left(\text { Assigned }_{p, q}\right)=1 \quad \text { where } p \in n, \\
& \quad \text { for } n=\{1,2,3, \ldots, N\}
\end{aligned}
$$

In this situation, at one time, a particular threat can be engaged by only one weapon. But a threat queue can also be made. If a threat is not successfully engaged, it is assigned to other suitable weapon.

Each weapon has start angle, sweep angle, range and other distinct parameters as shown in Table 1. The knowledge-base of threat provides useful information about these parameters. Here two aspects are given priority which include weapon's kp and threat's TI. Both these aspects contribute equally in the selection of weapons. The role of knowledge-base is to determine weapon characteristics, its state and $\mathrm{kp}$. However, the weapon state comprehends information such as working status, limitations and ammunition left. These parameters define weapon selection. Once a best threat-weapon pair is found; a proposal is sent to a selected weapon. At this stage selected weapon calculates its point of engagement (POE) with proposed threat. The weapons' knowledge-base rigorously employs weapon start, sweep angle and range for the calculation of POE. Next phase is to catch the best available aperture for proposed threat. This mechanism is valuable in real warfare situations; where multiple threats are impending in a formation. The procedure of finding time slot is analogous to time division multiplexing. If weapon is available during that POE time, it accepts the recommended threat for engagement otherwise threat is rescheduled on its next available niche. The next engagement niche is determined using numerous parameters. These parameters contain weapon ammunition left, stabilization time (stb), time to move (mov) from threat POE to another threat POE and reload time (rlt). The knowledge-base is used to provide weapon characteristic.

$$
\Delta t=\mathrm{rlt}+\mathrm{stb}+\mathrm{mov}
$$

If the designated weapon is busy and there is no available slot. Then the threat's proposal is directed to next best nominated 
weapon. This procedure continues until the threat is assigned. In this paper we are dealing with real warfare situation, where there are no constraints on weapons in term of number of threats. Only limitations is based on weapon physical system.

Weapon/ammunition qualification is also checked before assignment based on the following constraints:

(a) Suitable weapon/ammunition is out of stock.

(b) Suitable weapon/ammunition is obsoleted.

(c) Suitable weapon/ammunition is less in number in inventory.

Mathematically we expressed our weapon assignment model using the following decision variables:

$T_{i}>$ defined threshold value $\left(T_{\mathrm{v}}\right)$

$M_{i j}$ is the marriage parameter

$X_{i j t}$ is the threat $i$ assigned to weapon $j$ at the time slot $t$

We need to maximize

$$
\sum_{t=0}^{k} \sum_{j=0}^{m} \sum_{i=0}^{n} T_{i} M_{i j} X_{i j t}
$$

The variables (in Eq. 12) are $T_{i}, M_{i j}$ and $X_{i j t}$. The $T_{i}$ is a threat index value that is further based on multiple threshold value. Based on these values, weapons are assigned to approaching threat. The $M_{i j}$ is the variable that represents the marriage parameter. It maps and suggests the best possible cross matching of threat and weapon. It generates the alternatives based on the values of $T_{i}$ and $\mathrm{kp}$ of weapons. $X_{i j t}$ is the assignment or decision variable that assigns the threat $i$ to weapon $j$ at the time slot $t$ considering the constraints of inventory, supply chain and multiple engagements of weapons.

Maximization of number of weapons per threat and weapon constraints in case a threat is missed by an assigned weapon.

$$
\sum_{j=0}^{J} \sum_{t=0}^{T} X_{i j t} \leq W_{j}, \quad \forall j=0,1,2, \ldots, m
$$

Multiple weapon assignment to the threat that has TI value higher than the threshold value.

$$
\sum_{j=0}^{J} \sum_{t=0}^{T} X_{i j t} \geq M_{i j}, T_{i}>T_{v}
$$

A weapon to be assigned to one threat in one time slot.

$$
\sum_{i=0}^{m} X_{i j t} \leq 1, \quad \forall j=0,1,2, \ldots, n
$$

For making the model, more optimized and cost-effective, some other constraints are also applied in terms of weapon's availability in inventory and its supply from closest depot.

$$
\sum_{j=0}^{J} X_{i j t} \leq W_{j}, \quad \forall j=0,1,2, \ldots, m-q
$$

where $q \rightarrow$ weapon not available.

$$
W_{i}=f\left(\mathrm{TI}_{i}\right)+f\left(q_{d}\right)
$$

Availability of suitable weapon/ammunition in inventory check

WIC $=\left\{\begin{array}{ll}q=0 & \text { if } \quad w / a \text { is available in inventory } \\ q=m & \text { if } w / a \text { is not available in inventory } \\ q<m & \text { check amount of available } w / a\end{array}\right\}$

Weapon to be assigned to the threat in one time slot

$$
\sum_{j=0}^{J} X_{i j t} \leq 1, \quad \forall t=0,1,2, \ldots, p
$$

where $p$ is the total number of time slots.

$$
W\left(\text { assigned } \operatorname{slot}_{\mathrm{t}}\right)=\left\{\begin{array}{ll}
1 & \text { if slot is available } \\
0 & \text { Otherwise }
\end{array}\right\}
$$

While scheduling a weapon (WS), it is mandatory for weapon to accept the proposal.

$$
\mathrm{WS}_{i, j}= \begin{cases}1 & \text { if } \text { proposal }_{i, j}=1 \\ 0 & \text { Otherwise }\end{cases}
$$

Moreover, multiple engagements can also be made on single weapon as well as one threat is assigned to more than one weapon to ensure threat neutralization. If one weapon fails in engagement, then other weapon may succeed in neutralizing the threat. For multiple engagements, more constraints are added on DW and threats.

$$
\mathrm{DW}_{i}>T_{j}
$$

If load on DW increases the specific threshold LT, multiple engagement (ME) is difficult to obtain.

$$
\mathrm{ME}= \begin{cases}1 & \text { if } \mathrm{Ld} \leq \mathrm{LT} \\ 0 & \text { Otherwise }\end{cases}
$$

If a weapon is already assigned to threat, the status of weapon will be locked.

$$
W_{j}= \begin{cases}1 & \text { if not locked } \\ 0 & \text { Otherwise }\end{cases}
$$

While measuring the load on DW, multiple weapon assignment (MWA) is subject to:

$$
\text { MWA }= \begin{cases}1 & \text { if maximum assignment of } \mathrm{DW} \leq \mathrm{Ld} \text { of } \mathrm{DW} \\ 0 & \text { Otherwise }\end{cases}
$$


Table 2 Values showing kp of weapons using 3-D SMA

\begin{tabular}{|c|c|c|c|c|c|}
\hline & $W 1(\%)$ & $W 2(\%)$ & W3 (\%) & $W 4(\%)$ & W5 (\%) \\
\hline $\mathrm{T} 1$ & 50 & 51 & 63 & 54 & 74 \\
\hline $\mathrm{T} 2$ & 20 & 21 & 43 & 56 & 66 \\
\hline T3 & 76 & 9 & 15 & 59 & 89 \\
\hline $\mathrm{T} 4$ & 34 & 90 & 77 & 88 & 76 \\
\hline T5 & 12 & 8 & 53 & 93 & 69 \\
\hline T6 & 5 & 12 & 34 & 51 & 72 \\
\hline $\mathrm{T} 7$ & 78 & 10 & 47 & 18 & 12 \\
\hline $\mathrm{T} 8$ & 85 & 72 & 91 & 57 & 39 \\
\hline T9 & 73 & 31 & 47 & 69 & 88 \\
\hline $\mathrm{T} 10$ & 68 & 79 & 28 & 66 & 83 \\
\hline T11 & 57 & 96 & 41 & 75 & 13 \\
\hline
\end{tabular}

The 3-D SMA checks the compatibility of weapons to neutralize a particular threat and cross matches the threat with all DW that can potentially engage that threat. Succeeding the several parameters and constraints (as mentioned above), the $\mathrm{kp}$ values obtained varies from 0 to $100 \%$ as shown in the given matrix (Table 2). These values propose the threat-weapon mapping based on the $\mathrm{kp}$ of weapons. For example, to neutralize $\mathrm{T} 1$, there are 5 weapons deployed and the kill probability of these weapons are given in Table 2. It is observed that to neutralize T1, the best DW is W5 having 74\% kp for T1. If W5 is already engaged or having some other constraints (as mentioned above), the other option to neutralize T1 is W3. Similar is the case with other threats and weapons as shown in Table 2. Moreover, the TI and kp values are calculated after every $10 \mathrm{~s}$ to find the more accurate results. Therefore, these values keep on changing until the mission is accomplished.

In our proposed algorithm, we find launch entry and exit points of threat. In aerial threats, it is crucial to find launch points because in general weapon do not follow the straight path. Moreover, gravitational force and some other physical phenomena make a projectile motion (Naeem and Masood, 2010). Therefore, it is important to consider relative motion of a threat for successful neutralization. In such case, relative velocity and time can be helpful in finding more accurate decisions.

\section{Testing, analysis and validation}

\subsection{Testing of proposed approach}

To test our proposed approach, the modules were implemented. The command and control view shows the information of threats to commander and their approaching to weapons' range information to facilitate in decision making by highlighting the points at which threats can be hit by weapons. The problem is modelled as an assignment problem with a set of constraints. The mere modelling of the problem as a generalized assignment problem that can generate analytical results for any defined scenario. The model is validated through simulation. The values are computed using model and the same are used for simulation. The problem for two different scenarios is solved to get exact analytic results. These results are fed to the simulation engine for running the simulation. Therefore, in our case the analytical result are in agreement with the simulation results.

In test scenarios, the real-time warfare situation is maintained by deploying weapons covering the VA/VPs. Traditionally there is a one-to-one assignment among threats and weapons system as described in (Naeem et al, 2009). Flexibility of the proposed algorithm is also considered by creating many-to-many relationship model corresponding to the situation in factual battlefield. Initially we DW system to protect VA/VPs. Various combinations of weapons were placed to find the optimum deployment strategy.

For testing the proposed approach in real warfare scenario, the number of threats increased and MATLAB system allowed work under dynamic load. For bringing optimization, WA model was implemented as a separate server; which can integrate to real-time applications through interfacing module.

Scenario 1 In this scenarios, it is assumed that there are four threats, three defended assets and three DW. The TI values for all approaching threats are observed increasing with time except threat 1 , its output type varies from low to high and then again to low with TI $[0.1362, \ldots, 0.2132]$. Its values started decreasing after $30 \mathrm{~s}$, because the threat started moving in some other direction away from asset. The output type of threat 2 is increasing from low to very high with TI values $[0.2134, \ldots, 0.9110]$. Similarly the output type of threat 3 is ranked from very low to low $[0.0021, \ldots, 0.3123]$. The output type of threat 4 is ranked from low to high with value $[0.3214, \ldots, 0.6984]$ as shown in Figure 2a. Considering the rule of prioritizing the threats on the basis of their TI values (Dongfeng et al, 2012; Tanergüçlü et al, 2012), commander assigns the weapon 1 to threat 2 , the threat is hit by weapon at $35^{\text {th }}$ second. The weapon 2 is again assigned to threat 4 after $\Delta t$ because its $\mathrm{kp}$ is higher than other. The TI value of threat 4 is higher than other threats, hence weapon is 

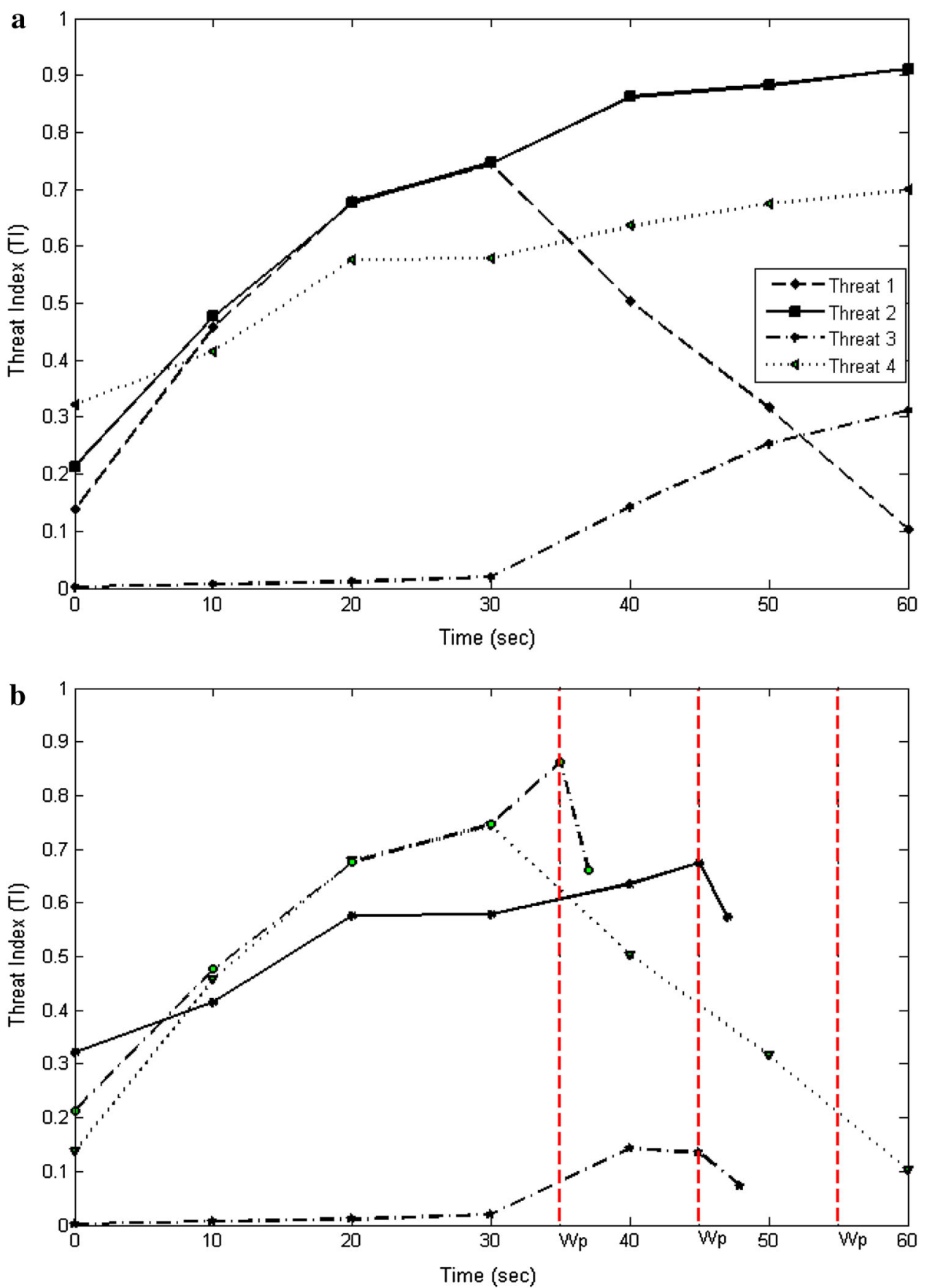

Figure 2 a TI of multiple approaching threats. b Threat neutralization by Weapon Assignment.

assigned to it. The threat 4 is neutralized around $45-48 \mathrm{~s}$. The threat 1 is not given due consideration, because it started moving in different direction and its TI decreased. Although threat 3 ranks risk to low, but even then, it is neutralized and hit by weapon to avoid high risk afterwards as shown in Figure $2 b$.

Scenario 2 The second scenario is more complex. It is assumed that there are four threats, two defended assets and four DW but it is observed that threats are appearing in different time intervals that may change the priority for threat neutralization, based on their TI values. The threat 1 is ranked from low to very high $[0.2432, \ldots, 0.7612]$, threat 2 high to very high with variations $[0.7122, \ldots, 0.8817]$, threat 3 very low to medium $[0.0121, \ldots, 0.5123]$ and threat 4 medium to very high $[0.4389, \ldots, 0.9710]$ as shown in Figure 3a. The threat 1 is appeared first, then threat 3 is appeared after $3 \mathrm{~s}$. The 

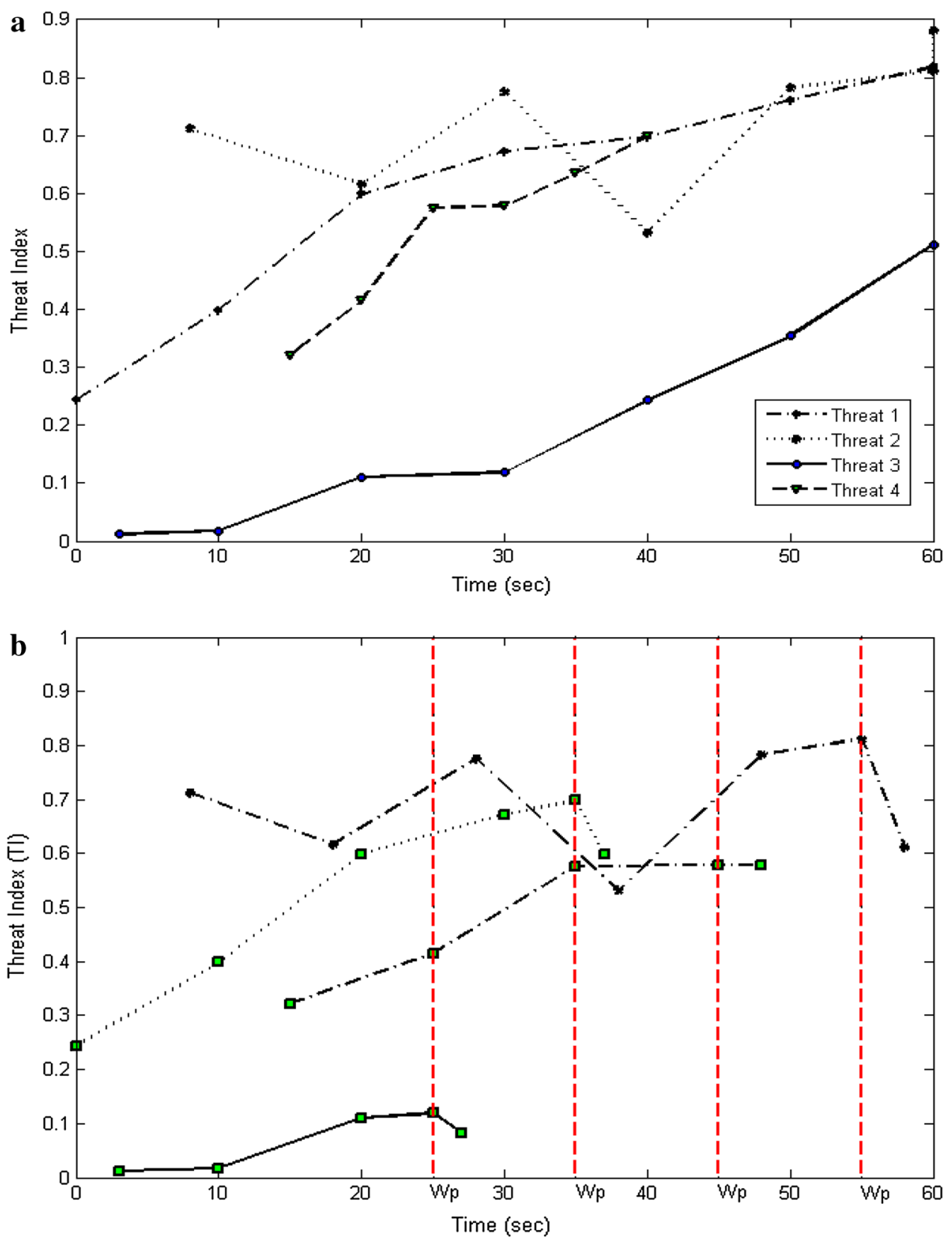

Figure 3 a TI of multiple approaching threats. b Threat neutralization by Weapon Assignment.

commander assigned weapon to threats. The threat 3 is in range of weapon, hence neutralized, while threat 1 is escaped because of its high speed. At 7th second, the threat 2 appears, its TI is higher than the other ones. But its value started decreasing. Meanwhile threat 4 appears and its value is increasing with time. The commander assigns the weapon to threat 1 with highest TI. The threat is hit by weapon. Then the weapon 2 is assigned to threat 4 because its TI is greater than threat 2, the weapon hits threat 4 and then again reloaded after $\Delta t$ and assigned to threat 2 , because its TI value is having variations and now increasing. Around 55-58 s, all approaching threats are neutralized with substantial decrease in their TI values and mission is accomplished as shown in Figure $3 \mathrm{~b}$.

\subsection{Analysis of proposed approach}

The scenarios were generated to test the optimality of implemented system. The simple SMA has already been compared with greedy algorithm and it was found that SMA algorithm produces more effective results as compared to greedy algorithm. One of the significant reason for the failure of greedy algorithm is that it focuses on minimum arrival time 

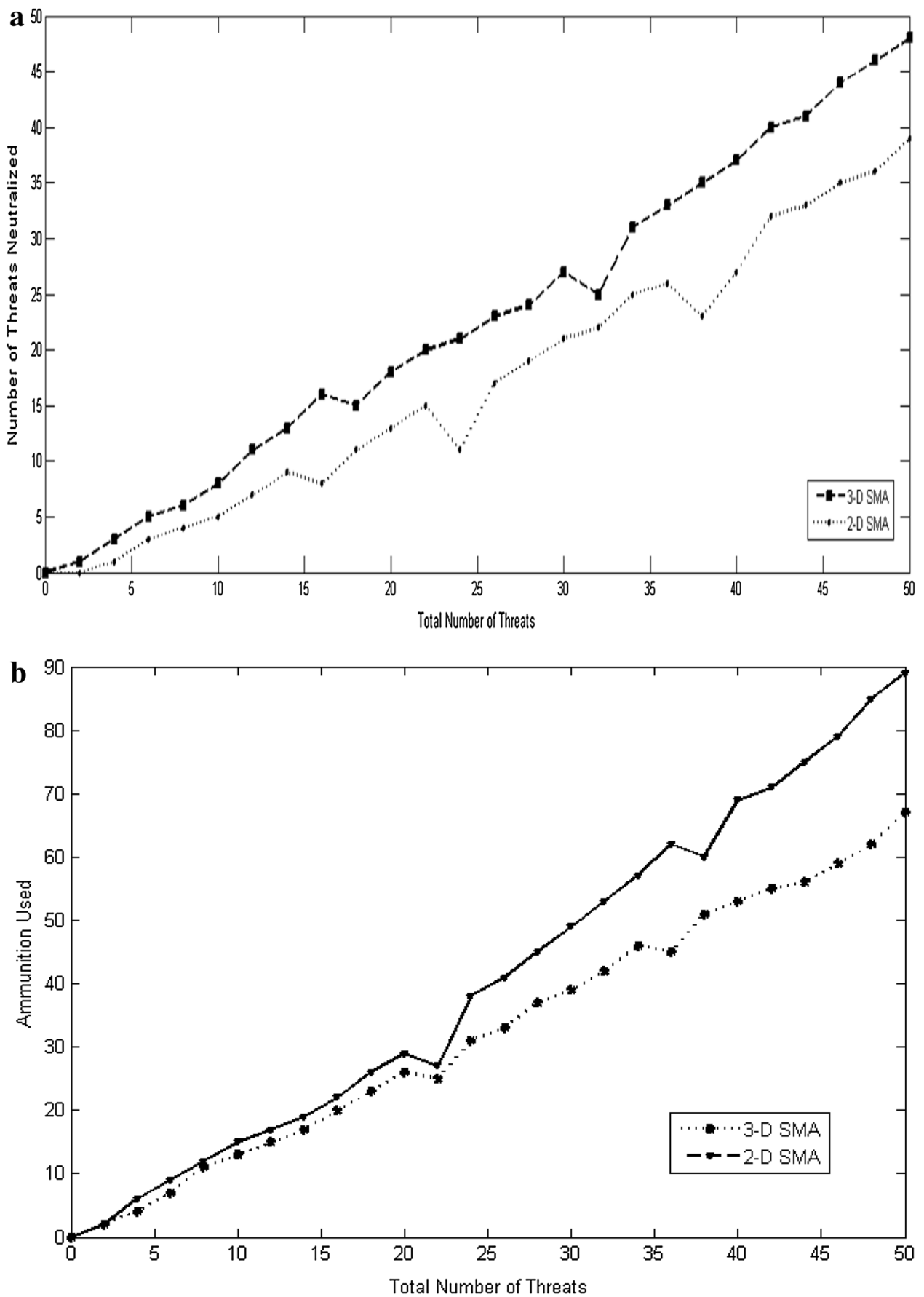

Figure 4 a Threat neutralization comparison. b Ammunition used comparison.

but does not consider the capability and intent parameters (Naeem and Masood, 2010). Therefore, we used SMA for comparison to our proposed system. The simple SMA uses the parameters of 2-D system and lacks the inventory check parameters in scheduling the weapons.

We perform testing of our system with various types of attack patterns, uncertainties, threat maneuvering and complexities were added. The complex situations were generated by increasing number of threats and decreasing number of deployed weapons. We perform comparison of previous proposed 2-D SMA on TEWA (Naeem and Masood, 2010) with our proposed 3-D SMA to measure its effectiveness. The number of threats neutralized by these algorithms were measured under the same circumstances and time frame. It has been found that threat neutralization is improved to greater extent i.e. $25 \%$ by using 3-D SMA in comparison with 2-D 


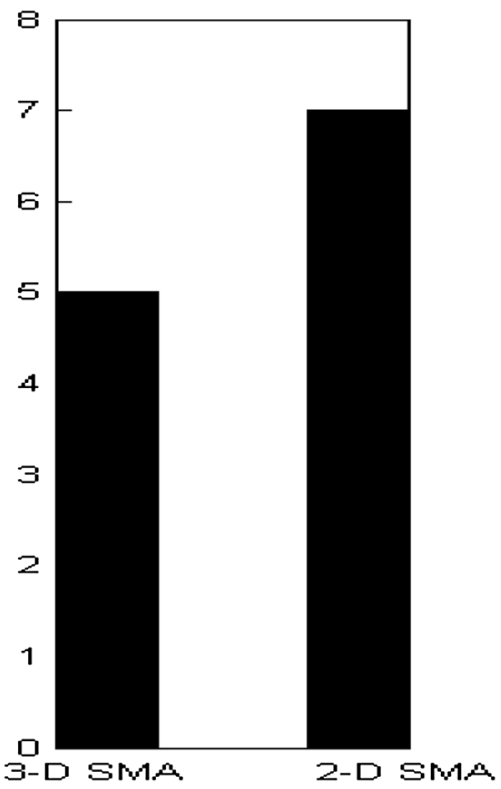

Figure 5 Damage asset comparison.

SMA (as shown in Figure 4a). Similarly, the amount of ammunitions used for threat neutralization were also recorded to compare and analyze the cost-effectiveness of the proposed algorithm. It is observed that amount of ammunition used is reduced to $31.1 \%$ in comparison with 2-D SMA because of more accurate values and calculations found using 3-D SMA (as shown in Figure 4b). If number of threats are increased, then amount of ammunition used in neutralization is also increased to manifold that may result in increase the cost of defense as well as in wastage of ammunition. It extends the statement that less amount of ammunition means reducing the defense cost.

The assets are likely to damage in defending them. The damage of assets was also calculated to validate the reliability of our proposed algorithm in terms of cost-effectiveness. It has been observed that the damage of assets is reduced to $28.5 \%$ as compared to 2-D SMA approach. The damage measurement was done based on capability index of threat, vulnerability index of asset and number of DW in that specific area (Naeem and Masood, 2010). One of the very problematic parameter, maneuvering was tested up to 55\% with our 3-D SMA while it was measured $40 \%$ in previous study (Naeem and Masood, 2010). With 3-D calculations, the results were found more accurate and precise in this aspect as well (as shown in Figure 5). Although proposed approach is much similar to 2-D SMA (Naeem and Masood, 2010), but 3-D parameters make it more effective in terms of reliability, efficiency and cost (in terms of ammunition usage and damage of assets) as well.

Although cost is not limited to only monetary terms when security is perilous factor. If the system provides cost-effective results, then it is more likely to adapt. Minimizing the defense cost (He and Zhuang, 2012) and maximizing the country's defense always remained an important factor for any country.
In our proposed approach, the cost means number of damages and ammunition usage in warfare. The cost of implementing this strategy is negligible, because it is upgradation of a system. Although, it may increase a little bit as compared to existing approaches, but it will assist in reducing losses and enhancing performance of the existing systems manifold with the integration of weapon's inventory and supply information in decision making process.

The key feature of this paper is to study the existing systems and propose a new approach augmenting the ability of the defense forces to impact the enemy's strategies. In practice, the measurable factors determining the threat's capability were its height, distance, speed, altitude, heading, formation, carrying weapons, lethality, radar-cross section, dive angle, maneuvering, attack approach, thermal images and heat signature in 2-D environment. While the weapon's capability was measured by its properties, range and $\mathrm{kp}$. Weapon was assigned on the basis of the information provided by these parameters. Our proposed approach makes addition of new parameters in the above mentioned information. The algorithm is extended for 3-D model and points of intersection of the 3-D target track with the semi sphere arc of fire of the weapon is computed. Our model not only provides 3-D information of threat's capability, but also assists in appropriate weapon selection and assignment using SMA. In this approach, a strong mathematical model is presented with the addition of new constraints that outperforms the previous approaches. In weapon selection, the ignored parameters (in previous studies) of inventory and supply are also encompassed to make TEWA more effective and efficient by increasing performance of threat neutralization with less ammunition and decreasing losses of assets in warfare environment. The model incorporates the $\mathrm{TI}$ along with the cross matching marriage score for optimization using SMA. It has been observed that the cost of asset damages were increased in previous approaches because the decision making process in TEWA lacks the few significant parameters and constraints that have been added in our approach. All of these properties of proposed 3-D SMA makes it unique, effective and innovative because unnecessary decision alternatives are automatically deleted that helps in making decisions quicker. Furthermore, our model will guide the government and defense forces to make their defense strategies and policies more effective considering the parameters and constraints mentioned in this paper.

\section{Conclusion and future research direction}

TE is a significant component in military and non-military applications. Miscalculations in threat assessment could lead to unprecedented damages. TE not only helps in weapon assignment, but it enables commanders to understand the behavior of enemy tactics. In this context, a novel 3-D SMA is proposed for TEWA that clearly indicates the usefulness of new variables identified in the model. It is worth mentioning 
that existing literature on TEWA lack this information and therefore open very interesting research problem that we tackled through our proposed algorithm. Thus, our model has made an important contribution in making existing system more effective and efficient. We further believe that at most occasions, research done on TEWA has emphasized on known threat types with less focus on real warfare scenario that is a challenging task. In warfare situations, where sensors play a vital role in threat identification, there are several other parameters to evaluate the threats. In this study, we designed and implemented an efficient real time TE and WA system based on knowledge-base system and 3-D SMA. The knowledge-base is intelligently updated based on result of weapon assignment and 3-D SMA encompasses the parameters that are essential for aerial threats. To check the validation of the proposed model, we performed simulations using real-time $\mathrm{AD}$ scenarios and observed the outcomes. Moreover, we performed comparison of our proposed approach with existing ones. The results show the accuracy, correctness, minimum errors and reliability in the proposed system. Hence, it can be stated that this system will bring a significant contribution in this field.

\section{References}

Allouche MK (2005). Real-time use of Kohonen's self-organizing maps for threat stabilization. ScienceDirect: Information Fusion 6(2):153-163.

Azimirad E and Haddadnia J (2015). Target threat assessment using fuzzy sets theory. Advances in Intelligent Information 1(2):57-74.

Barkdoll TC, Gaver DP, Glazebrook KD, Jacobs PA and Posadas S (2002). Suppression of enemy air defenses (SEAD) as an information duel. Naval Research Logistics 49(8):723-742.

Beare GC (1987). Linear programming in air defence modelling. Journal of Operational Research Society 38(10):899-905.

Cavalcante CAV, Ferreira RJP and Almeida AT (2010). A preventive maintenance decision model based on multicriteria method PROMETHEE II integrated with Bayesian approach. IMA Journal of Management Mathematics 21(4):333-348.

Choi BJ, Kim JE, Kim JS and Kim CO (2016). Fuzzy rule-based method for air threat evaluation. Journal of the KIMST 19(1):57-65.

Dongfeng C, Yu F and Yongxue L (2012). Threat assessment for air defense operations based on intuitionistic fuzzy logic. In: International workshop on information and electronics engineering (IWIEE), procedia engineering (Vol. 29, pp. 3302-3306).

Erlandsson T and Niklasson L (2013). A five states survivability model for missions with ground-to-air threats. In SPIE Proceedings of modeling and simulation for defense systems and applications (Vol. 8752).

Erlandsson T and Niklasson L (2014). Automatic evaluation of air mission routes with respect to combat survival. Information Fusion 20(1):88-98. doi:10.1016/j.inffus.2013.12.001

French S (2015). Cynefin: uncertainty, small worlds and scenarios. Journal of the Operational Research Society 66(10):1635-1645.

Govindan K, Soleimani H and Kannan D (2015). Reverse logistics and closed-loop supply chain: A comprehensive review to explore the future. European Journal of Operational Research 240(3):603-626.
Hausken K and Zhuang J (2012). The timing and deterrence of terrorists attacks due to exogenous dynamics. Journal of the Operational Research Society 63(6):726-735.

He F and Zhuang J (2012). Modelling 'contracts' between a terrorist group and a government in a sequential game. Journal of the Operational Research Society 63(6):790-809.

Hossein PA and Athans M (1990). Preferential defense strategies, Part 1: The static case.

Hosein PA, Walton JT and Athans M (1988). Dynamic weapon target assignment problem with vulnerable $\mathrm{C} 2$ nodes. Massachusetts Institute of Technology Cambridge Lab for Information and Decision Systems.

Iwama K (2008). A survey of the stable marriage problem and its variants. In Proceedings of the international conference on informatics education and research for knowledge-circulating society.

Johansson F and Falkman G (2008). A Bayesian network approach to threat evaluation with application to an air defense scenario. In: information fusion, 2008 11th international conference, pp. 1-7.

Johansson F and Falkman G (2008). A comparison between two approaches to threat evaluation in an air defense scenario. In Proceedings of the 5th international conference on modeling decisions for artificial intelligence, October, Sabadell, Spain, Springer, pp. 110-121.

Johnston W, Quigley J and Wall L (2006). Optimal allocation of reliability tasks to mitigate faults during system development. IMA Journal of Management Mathematics 17(2):159-169.

Karasakal O (2008). Air defense missile-target allocation models for a naval task group. Computers and Operations Research 35(6): $1759-1770$.

Kirby M and Capey R (1997). The air defence of Great Britain, 1920-1940: An operational research perspective. Journal of the Operational Research Society 48(6):555-568.

Laird CL (2016). Heterogeneous defensive naval weapon assignment to swarming threats in real time. Thesis submitted to Monterey. Monterey, CA: Naval Postgraduate School.

Li N, Yi W, Sun M and Gong G (2012). Development and application of intelligent system modelling and simulation platform. Simulation Modelling Practice and Theory 29:149-162.

Liao S (2000). Case-based decision support system: Architecture for simulating military command and control. European Journal of Operational Research 123(3):558-567.

Liebhaber M and Feher B (2002). Air threat assessment: Research, model and display guidelines. In Proceedings of command and control research and technology symposium (pp. 1-15).

Lotter DA, Nieuwoudt I and Vuuren JHV (2013). A multiobjective approach towards weapon assignment in a ground-based air defence environment. Orion 29(1):31-54.

Lozano JMS, Serna J. and Payan AD (2015). Evaluating military training aircrafts through the combination of multi-criteria decision making processes with fuzzy logic. A case study in the Spanish Air Force Academy. Aerospace Science and Technology 42:58-65.

Mardani A, Jusoh A and Zavadska EK (2015). Fuzzy multiple criteria decision making techniques and application-Two decades review from 1994 to 2014. Expert System with Applications 42(8): 4126-4148.

Masud ASM, Metcalf P and Hommertzheim D (1995). A knowledgebased model management system for aircraft survivability analysis. European Journal of Operational Research 84(1):47-59.

Naeem H, Masood H, Mukhtar H and Khan SA (2009). A novel twostaged decision support based threat evaluation and weapon assignment algorithm, asset-based dynamic weapon scheduling using artificial intelligence techniques. International Journal of Computer Science and Information Security (IJCSIS). 
Naeem H and Masood A (2010). An optimal dynamic threat evaluation and weapon scheduling technique, Knowledge-Based Systems 23(4):337-342.

Nguyen BU, Smith PA and Nguyen D (1997). An engagement model to optimize defense against a multiple attack assuming perfect kill assessment. Naval Research Logistics 44(7):687-697.

Nguyen XT (2002). Threat assessment in tactical airborne environments. In: Proceedings of the 5th international conference on information fusion 2:1300-1307.

Onay O (2016). A mathematical approach to neuromarketing: A weapon-target assignment model. International Journal of Academic Research in Business and Social Sciences 6(1):164-173.

Oxenham MG (2003). Enhancing situation awareness for air defence via automated threat analysis. Information Fusion, 2003. In: Proceedings of the sixth international conference (Vol. 2, pp. 1086-1093).

Paulson EC, Linkov I and KeislerJM (2016). A game theoretic model for resource allocation among countermeasures with multiple attributes. European Journal of Operational Research 252(2): 610-622.

Paradis A, Oxenham, M and Cutler P (2005). Threat evaluation and weapons allocation in network-centric warfare. In 8th International conference on information fusion, Canada.

Phillips-Wren G, Mora M, Forgionne GA and Gupta JND (2009). An integrative evaluation framework for intelligent decision support systems. European Journal of Operational Research 195(3): 642-652.

Riveiro M, Helldin T, Lebram M and Falkman G (2013). Towards future threat evaluation systems: User study, proposal and precepts for design. In: 16th international conference on information fusion, Istanbul, Turkey (pp. 1863-1870).

Riveiro M, Helldin T, Falkman G and Lebram M (2014). Effects of visualizing uncertainty on decision-making in a target identification scenario. Computers \& Graphics 41:84-98.

Roy J, Paradis S and Allouche M (2002). Threat evaluation for impact assessment situation analysis systems. In Proceedings of (SPIE), Signal Processing, Sensor Fusion, and Target Recognition, Orlando (Vol. 4729, p. 13).

Roux JN and Vuuren JH (2007). Threat evaluation and weapon assignment decision support: A review of the state of the art. ORiON: The Journal of ORSSA 23(2):151-187.
Sahin MA, Leblebicioglu K (2014). Approximating the optimal mapping for weapon target assignment by fuzzy reasoning. Information Sciences 255:30-44.

Sahoo R (2016). Weapons of mass destruction in West Asia and its massive influence in Iran: An analysis. Ars Artium: An International Peer Reviewed-cum-Refereed Research Journal of Humanities and Social Sciences 159-166.

Shan X, and Zhuang J (2013). Hybrid defensive resource allocations in the face of partially strategic attackers in a sequential defenderattacker game. European Journal of Operational Research 228(1):262-272.

Shan X and Zhuang J (2014). Subsidizing to disrupt terrorism supply chain-A four player sequential game. Journal of the Operational Research Society 65(7):1108-1119.

Smith M, Dekker R, Kos J and Hontelez J (2001). The availability of unmanned air vehicles: a post-case study. Journal of the Operational Research Society 52(2):161-168.

Tanergüçlü T, Maraş H, Gencer C and Aygüneş H (2012). A decision support system for locating weapon and radar positions in stationary point air defense. Infusion System Front 14(2): 423-444. 10.1007/s10796-010-9269-6.

Truter ML (2016). Development and demonstration of a performance evaluation framework for threat evaluation and weapon assignment systems. Thesis presented in the Faculty of Engineering at Stellenbosch University.

Yıldırım UZ, Tansel BÇ and Sabuncuoğlu I (2009). A multi-modal discrete-event simulation model for military deployment. Simulation Modelling Practice and Theory 17(4):597-611.

Wu K and Ye S (2014). An information security threat assessment model based on Bayesian network and OWA operator. An International Journal of Applied Mathematics and Information Sciences 8(2):833-838.

Received 16 February 2016; accepted 6 October 2016 